\title{
L'image des organisations humanitaires à l'épreuve des pratiques de communication : vers des structures hybrides
}

\section{Stéphanie Dilliere-Brooks}

\section{(2) OpenEdition}

\section{Journals}

Édition électronique

URL : http://journals.openedition.org/communicationorganisation/557

DOI : 10.4000/communicationorganisation.557

ISSN : 1775-3546

Éditeur

Presses universitaires de Bordeaux

Édition imprimée

Date de publication : 1 décembre 2008

Pagination : 24-37

ISBN : 978-2-86781-582-9

ISSN : 1168-5549

\section{Référence électronique}

Stéphanie Dilliere-Brooks, « L'image des organisations humanitaires à l'épreuve des pratiques de communication : vers des structures hybrides », Communication et organisation [En ligne], 34 | 2008, mis en ligne le 01 décembre 2011, consulté le 10 décembre 2020. URL : http://

journals.openedition.org/communicationorganisation/557 ; DOI : https://doi.org/10.4000/ communicationorganisation.557 


\title{
Dossier : Retour sur les images d'organisations
}

\section{Résumé}

L'image de l'organisation est devenue pour les acteurs humanitaires un enjeu de taille. Afin d'assurer leur survie et leur stabilité dans un contexte international de plus en plus questionnant, les organisations de l'aide internationale ont développé, à travers des pratiques de communication souvent empruntées au service marchand, des images valorisantes à destination de leurs parties prenantes. Dans ses deux assertions, l'image fabriquée et diffusée à l'extérieur, comme celle relative à la perception des membres de ce que leur environnement perçoit de leur institution, cette étude empirique menée auprès de quatre grandes organisations humanitaires francophones, tend à comprendre non seulement les configurations de ces deux types d'images mais aussi leurs relations et impacts sur les concepts de légitimité et d'identité.

\section{Mots-clefs}

Organisations humanitaires, légitimité, légitimation, identité organisationnelle, image organisationnelle, communication syntone, communication dissonante.

\begin{abstract}
Image increasingly assumes an important issue for humanitarian organizations. With the aim of assuring their survival, as much as their stability, in an increasingly challenging world, they have developed, through several communications approaches adapted from the private sector, imaging design specifically for their stakeholders. Such imaging has two forms in humanitarian organizations today: that which is purposed-designed for specific target groups, and that derived from the perception of organizational members concerning their specific environment. This empirical study, led within four leading humanitarian organizations, will attempt to draw conclusions about the relationships between imaging and key concepts of legitimacy and identity.
\end{abstract}

\section{Key-Words}

Humanitarian organisations, legitimacy, legitimation, organisational identity, organisational image, dissonant communication, congruent communication.

Stéphanie Dillière-Brooks est docteur en Sciences de l'Information et de la Communication de l'Université Michel de Montaigne - Bordeaux 3. Elle a été professionnelle du terrain humanitaire pendant de nombreuses années. Le secteur humanitaire aux prises avec le questionnement de sa légitimité et de son identité reste son principal thème de recherche. 
L'image des organisations humanitaires...

\title{
L'image des organisations humanitaires à l'épreuve des pratiques de communication : vers des structures hybrides
}

\author{
Stéphanie Dillière-Brooks
}

dillierebrooks@gmail.com

L'image organisationnelle est une notion protéiforme difficilement saisissable même si elle semble centrale, voire incontournable dans les préoccupations institutionnelles. Elle est l'interface voulue ou parfois subie existant entre l'organisation et son environnement autant que le fruit des échanges intra-organisationnels. Dans une société marquée par la suprématie de l'image, on ne sera pas surpris de voir que tous les secteurs sont maintenant soumis à ce dogme et tentent, par des stratégies de communication, de jouer un rôle plus proactif et plus déterminant sur les configurations possibles de leurs représentations.

Les acteurs humanitaires n'échappent pas à cette règle. Depuis, les années 90, la communication, jusqu'alors taboue, est devenue une ressource principale, incarnée par la mise en place de véritables directions de la communication et d'outils plus performants et plus pointus pour attirer l'attention des publics. Désireuses d'être connues et reconnues autant que de bénéficier d'une perception positive de la part de leur environnement, ces institutions humanitaires envisagent cette course à l'image comme un « mal nécessaire ».

Pour mieux en saisir la dimension stratégique, il est important de tenter de définir les relations que cette image entretient, à travers ces pratiques communicationnelles, avec deux autres notions toutes aussi omniprésentes : la légitimité et l'identité. Quel phénomène semble pouvoir lier ces trois paramètres et quels en sont les mécanismes régulateurs, autant que déstabilisateurs?

Pour essayer d'y répondre, cette étude empirique propose d'effectuer une étude de cas de quatre organisations humanitaires européennes francophones en mixant différents outils de recherche (Giroux, 2003) ${ }^{1 .}$ Les institutions retenues sont le Comité International de la Croix-

1 N. Giroux, L'étude de cas, Conduire un projet de recherche - une perspective qualitative, Y. Giordano (sous dir.), Colombelles, Editions EMS, 2003, c'est-à-dire 42-84. 


\section{Dossier : Retour sur les images d'organisations}

Rouge (CICR, Suisse), Médecins Sans Frontières (MSF, France), Action Contre la Faim (ACF, France) et Handicap International (HI, Belgique). Après avoir clarifié les concepts concernés grâce à une revue de la littérature, les pratiques communicationnelles, initiatrices de l'image volontairement diffusée, ont été analysées. Cela a été effectué par un recensement des pratiques et outils élaborés par les directions de la communication. Ils ont été, dans un second temps, confrontés aux propos de 10 acteurs sélectionnés selon la méthode non probabiliste (Dépelteau, 2002) ${ }^{2}$ dans chaque structure. Ils ont été interrogés via des questions ouvertes sur leurs perceptions de cette image et de cette communication. Dans le cadre d'une perspective constructiviste selon laquelle les flux et processus de communication donnent du sens à l'organisation tout autant que les commentaires faits à leur sujet, une analyse de contenu d'abord intrinsèque à chaque structure puis transversale a été menée. Cette confrontation semble avoir montré que les pratiques communicationnelles sont soit orientées sur un effort de légitimation, générant une image parcellaire, soit focalisées sur l'identité reléguant l'image au second plan des préoccupations des acteurs.

\section{L'image organisationnelle : entre fabrication et perception}

Le concept d'image est à la fois omniprésent et malléable au sein des organisations. Les tentatives de définition et de clarification ont concentré toutes les attentions non seulement des chercheurs en sciences de l'information et de la communication, mais aussi de ceux attachés aux sciences de gestion et au marketing, notamment outreAtlantique. Plusieurs formes en ont été proposées. La première élaborée par Gilles Marion (1989; p. 23$)^{3}$ est une conception triptyque faisant coexister l'image déposée, représentation collective des parties prenantes de l'entreprise, l'image voulue, symbole des valeurs déclarées par le noyau stratégique, avec l'image diffusée comme discours tenu par et sur l'entreprise. Dès lors, on note que cette notion peut être à la fois l'expression d'une volonté institutionnelle à destination de l'extérieur, tout comme le résultat de la perception de l'organisation par son environnement direct.

Ces deux acceptions, mêlant une perception active et un ressenti réactif, agissent comme des leitmotive à travers les différentes

2 F. Dépelteau, La démarche d'une recherche en sciences humaines, Laval, Les Presses de l'Université de Laval, 2002, p. 214.

3 Marion G., Les images de l'entreprise, Paris, Les Editions de l'Organisation, 1989. 
définitions et réflexions menées sur ce thème. Comme le précise Albert E. Whetten (2006, p : 228) ${ }^{4}$, l'image est caractérisée à la fois comme la représentation projetée de l'organisation vers les parties prenantes extérieures ciblées et comme la représentation de l'organisation exprimée par ces mêmes acteurs, semblable à la réputation.

Cela nous éclaire sur sa direction, c'est-à-dire sur les relations de l'organisation vers son environnement comme à l'inverse de l'environnement vers l'organisation. Deux autres paramètres semblent pouvoir encore être soulignés. Pour certains, l'image est le résultat d'une construction communicationnelle volontaire de l'organisation à destination de son environnement (Kapferer, 1988, p : 76) ${ }^{5}$, et donc synonyme « (...) de la façon dont le public décode l'ensemble des signes provenant des produits, services, publicités ». Pour d'autres (Dutton, Dukerich et Harquail, 1994 ; Pratt et Foreman, 20007), elle peut aussi être présentée comme le reflet du ressenti des acteurs internes à l'encontre de ce que leur environnement pense de leur structure d'appartenance. Dès lors, on note deux niveaux de configuration de cette image jouant, à la façon d'un miroir, entre les perceptions des parties prenantes externes à l'organisation et celles des protagonistes internes.

Sans céder à la facilité d'offrir une vision dichotomique de l'image interne et externe, nous avons mis cependant en relation l'image fabriquée et communiquée vers la société, résultat d'un processus proactif, avec l'image perçue de cette dernière par les acteurs clés internes, comme produit réactif. Dans cette vision de l'organisation, l'image est donc plurielle mais garde un soubassement commun : la communication.

\footnotetext{
${ }^{4}$ Whetten A.E., Albert and Whetten revisited-Strenghtening the concept of organizational identity, Journal of Management Inquiry, Vol. 15, $\mathrm{n}^{\circ} 3$, September 2006, pp. 219-234.

5 Kapferer J-N., Maitriser l'image de l'entreprise : le prisme de l'identité, Revue Française de Gestion, Nov-Déc. 1988, pp : 78-82.

6 Dutton J.E., Dukerich J.M., Harquail C.V., Organizational images and member identification, Administrative Sciences Quarterly, 39, 1994, c'est-àdire 239-263.

7 Pratt M.G., Foreman P.O:, Classifying Managerial responses to multiple Organizational identities, Academy of Management Review, vol. 25, $\mathrm{n}^{\circ} 1$, 2000, c'est-à-dire 18-42.
} 
Dossier : Retour sur les images d'organisations

\section{Les pratiques communicationnelles comme fondements}

Dans toutes les définitions développées préalablement, force est de constater la présence de la communication non seulement comme outil de diffusion de l'image, mais aussi comme support de son retour en direction des acteurs internes. Elle est le résultat d'une confrontation à différents niveaux de l'organisation et de son environnement. De même, la communication, comme mise en commun de l'information (Ardoino, Paulré, 1993) ${ }^{8}$ tout comme transaction par laquelle les partenaires bâtissent leur relation et construisent l'organisation (Giroux, 1994 ${ }^{9}$, revêt des formes différentes. Elle est institutionnalisée à travers ses stratégies, ses pratiques et ses outils mis en place, mais elle peut aussi se manifester sous «la forme d'évènements communicationnels non prescrits, non prévisibles, contingents » (Bernard, 1998, p. 48) ${ }^{10}$ voire être constituée des échanges comme des discours informels générés par les acteurs au fur et à mesure de l'expérience.

De façon pragmatique, les pratiques de visibilité comme la charte visuelle, les campagnes institutionnelles déclinées sur tous les supports, et les actions de lisibilité comme les relations presse, les évènements de sensibilisation, et de mécénat sont autant de procédures proactives destinées à concourir à l'obtention d'une image auprès des publics directs autant qu'indirects. Parallèlement, les systèmes de veille comme les centres de recherche, de documentation, d'étude de la concurrence, ou simplement tous les outils d'observation de l'opinion publique (ex: les sondages) semblent favoriser la constitution de la perception des membres de ce que les acteurs extérieurs pensent de leur organisation.

Ces deux mouvements ne vont pas sans l'intervention, parfois impromptue, de processus d'échanges informels et involontaires qui ont, très souvent, un impact important et parfois distordant sur l'image

8 Ardoino J., Paulré B., Groupes, relations et communication, Dictionnaire Critique de la Communication (sous dir.) L. Sfez, Tome 1, Paris, Puf, 1991, c'est-à-dire 602-618.

9 Giroux N., La communication interne : une définition en évolution, Communication \& Organisation $\mathrm{n}^{\circ} 5$, La communication interne: une approche croisée, GREC/O, 1994.

10 Bernard F., Communication organisationnelle et usages des sciences humaines et sociales. Fragments de réflexion. Communication d'entreprises et d'organisations (textes réunis par C. Le Moenne), Rennes, PUR, 1998, pp : 48-73. 
diffusée aux parties prenantes comme sur la représentation que s'en font les acteurs internes.

La communication est, a priori, le support essentiel favorisant la constitution des images de l'organisation et semble en présenter les mêmes caractéristiques : c'est une construction permanente et sans cesse renouvelée. Elle comporte des aspects voulus tout autant qu'involontaires voire subis. Elle est à double sens allant de l'organisation vers son environnement et vice versa. Mais, ces points communs semblent pouvoir établir un lien avec deux autres processus à l'œuvre au sein des institutions, issus aussi des pratiques communicationnelles mises en place.

\section{L'image comme enjeu des processus de légitimation et de construction identitaire.}

L'objet organisationnel est complexe d'autant plus dans une société multiforme où les acteurs foisonnent et doivent définir et sauvegarder leur espace et leur positionnement. L'image, à travers l'avènement des médias, prédomine et chacun, à l'échelle individuelle comme organisationnelle, semble devoir affirmer comme justifier son existence, ses actions à travers la grille médiatique dorénavant imposée. Cela semble tisser le premier lien entre cette image et le concept de légitimité.

Définie par Mark C. Suchman (1995, p. 574) ${ }^{11}$ comme «une perception ou une représentation généralisée selon laquelle les actions d'une entité sont désirables, justes ou appropriées, à l'intérieur d'un certain système socialement construit de normes, de valeurs, de croyances et de définitions ", cette légitimité semble particulièrement stratégique dans une période de profonde remise en question planétaire. En effet, c'est comme si les organisations, aux prises avec une crise généralisée du lien social, avaient de plus en plus besoin de justifier leur droit d'exister (Maurer, 1971, p. 361) ) $^{12}$ à travers des actions de légitimation, en grande partie communicationnelles. Cela ne semble pas pouvoir aller sans la diffusion d'une certaine image aux contours avantageux, au contenu valorisant l'essence autant que les actions de la structure. On l'aura compris, l'image, au sens de ce qui

11 Suchman M., Managing Legitimacy: Strategic and Institutional Approaches, Academy of Management Review, vol. 20, ํ3, 1995, pp. 571610.

12 Maurer J.G., Readings in organizational theory: open-system approaches, New York, random House, 1971. 


\section{Dossier : Retour sur les images d'organisations}

est diffusé à l'extérieur, semble être un élément facilitateur voire un support incontournable dans le processus de la légitimation auprès de différents publics.

Ses frontières semblent être plus floues avec la notion d'identité organisationnelle. En effet, l'identité est présentée comme le résultat de ce qui est vu par les acteurs internes comme étant les caractéristiques centrales, distinctives et continues de leur organisation (Albert et Whetten, 1985, 2006) ${ }^{13 .}$ Alors que cette identité se construit à travers les échanges et donc obéit à un processus sans fin de coconstruction, les influences de l'image sont conséquentes à deux titres. Tout d'abord, l'image, au sens de la croyance des membres de ce que l'environnement pense de leur organisation, génère une comparaison inévitable avec l'identité. Cela ne peut que provoquer des ajustements et des modifications car, finalement, les membres affirment comment ils voient leur organisation en fonction de comment ils pensent que les acteurs extérieurs la voient. A ce stade, ils ont le sens de la divergence (l'incohérence) ou de l'alignement de leur identité au regard de cette image (Corley, Gioia, 2004) ${ }^{14}$ Ce lien est même présenté sous forme de triangulation par Pierre Legendre (1994, p: 67) ${ }^{15}$. Selon lui, l'identité repose «sur le sujet et son image mais aussi sur le miroir instaurateur de cette division ». La communication semble y prendre toute son ampleur car elle peut orienter cette relation et favoriser sa divergence autant que sa congruence.

Ensuite, ses influences sont conséquentes dans un contexte où l'image à destination de l'extérieur est sacralisée et souvent formulée en priorité aux dépens d'une identité mal définie (Kapferer, 1988). Cela l'affaiblit et peut favoriser une dichotomie entre les pratiques communicationnelles et l'identité organisationnelle. L'image semble donc avoir un lien fort avec les concepts de légitimité et d'identité. Dans une certaine mesure facilitatrice de la légitimation quand elle est diffusée à l'extérieur, elle peut néanmoins déstabiliser l'identité lorsqu'elle dissone en interne. Au regard de la littérature, il semble y

13 Albert S., Whetten D.A., Organizational Identity, Research in Organizational Behaviour, Vol. 7, L.L. Cummings \& B.M. Staw, Eds Greenwich, CT, JAI, 1985, pp. 263-295.

14 Corley K.G., Gioia D.A., Identity Ambiguity and Change in the Wake of a corporate spin-off, Administrative Science Quarterly, n49, 2004, pp. 173208.

15 Legendre P., Leçon III- Dieu au miroir, Etude sur l'institution des images, Paris, Fayard, 1994. 
avoir un mécanisme corrélatif mais le lien établi à travers ces processus communicationnels n'est pas encore prouvé notamment si l'on veut y intégrer l'élément nouveau et déterminant qu'est la légitimité. Pour cela, une étude empirique dans un secteur particulier et exemplaire est proposée.

\section{Le secteur humanitaire aux prises avec son image}

Le secteur humanitaire, depuis le début des années 90 , semble défié et questionné par son environnement pour des raisons conjoncturelles multiples: la fin de la guerre froide, l'apparition de la lutte antiterroriste et l'émergence de conflits plus nombreux et plus complexes entre autres. Cela est dédoublé par des causes structurelles comme la professionnalisation du secteur, son institutionnalisation et son homogénéisation. Ces deux mutations vont de pair et s'autoalimentent l'une l'autre. Mais, ce qui marque principalement ce champ, c'est le foisonnement des acteurs rendant plus difficile leur différenciation et la sauvegarde de leur espace respectif en même temps que leur médiatisation accrue ce qui change considérablement les configurations de structures jusqu'alors travaillant dans l'ombre. Ces deux phénomènes sont inextricables. Ces structures découvrent, peu à peu, l'importance de l'image, vitale à la continuation de leurs actions. En effet, cela peut faciliter l'accès à certains terrains, aider à l'obtention de fonds d'acteurs privés autant que publics et générer une augmentation de leur crédibilité nécessaire à la dénonciation des crises oubliées. Pour ces organisations humanitaires modernes, l'image est clairement un outil de légitimation susceptible de les aider à continuer à exister dans cet environnement mouvant et questionnant. Dès lors, deux tendances se dessinent dans cette course à l'image. D'une part, on assiste à la structuration de la communication au sein des organisations avec de véritables départements rattachés aux directions générales. D'autre part, il y a une multiplication des actions de visibilité et de lisibilité dans leur pays d'origine comme d'intervention. C'est un fait nouveau et qui, par son ampleur, ne va pas sans heurts. Cela oppose les volontaires humanitaires de la première heure aux nouveaux gestionnaires, soucieux d'améliorer la réputation, la reconnaissance et la légitimité à travers une image travaillée.

Cet article propose de rendre compte des résultats les plus significatifs et éclairants pour notre postulat théorique. 


\section{Dossier : Retour sur les images d'organisations}

\section{Vers deux tendances communicationnelles...}

$\mathrm{Au}$ regard de la confrontation des pratiques communicationnelles mises en œuvre et des propos des acteurs internes, il est intéressant de soulever l'apparition de certaines similitudes transversales comme de quelques divergences, éléments d'ailleurs particulièrement révélateurs pour cette étude.

Tout d'abord, quel que soit leur profil hiérarchique ou professionnel, les acteurs interrogés ont unanimement mis en avant un problème d'image auprès de l'opinion publique. Les arguments avancés sont une mauvaise connaissance du secteur humanitaire en général. Il y a une grande confusion dans l'esprit du citoyen, récipiendaire d'une information souvent partielle et tributaire d'un agenda médiatique déjà très chargé.

Ils s'accordent tous aussi à souligner les difficultés croissantes, ces dernières années, en termes de remise en question de leur espace jusqu'alors imparti, et de l'empiètement de leurs valeurs par un certain nombre d'acteurs non humanitaires. Ils éprouvent un véritable questionnement quant à leur légitimité et, pour cela, abordent les nouvelles politiques de communication comme une nécessité. Mais, ils continuent à les craindre car elles risquent, en étant trop prédominantes, de déstabiliser le ratio d'activités entre les opérations et les communications, ou plus exactement entre les frais directement destinés à la victime et ceux alloués au fonctionnement de la structure et donc à sa reproduction.

Par contre, très rapidement et spontanément, deux tendances semblent se distinguer; chacune caractérise un groupe de deux organisations. En effet, on note que les perceptions de ces pratiques de communication et de l'image en provenance de l'extérieur sont à nuancer.

\section{Vers une communication syntone}

Pour certaines, comme le CICR et MSF France, la confrontation des perceptions des acteurs avec les pratiques réellement en œuvre, a mené à plusieurs résultats. Même si l'image est vue comme un peu nébuleuse dans l'esprit des citoyens en général, on note dans la grande majorité des propos une certaine confiance en la reconnaissance spontanée de l'organisation, de son logo, de son label et en une appréciation, dans l'ensemble positive, de l'opinion publique. Cela est renforcé par une bonne connaissance de la structure par les donateurs privés, principalement dans le cas de MSF financée à $99 \%$ par eux. Dans les faits, cette image diffusée à l'extérieur est d'ailleurs très 
fortement attachée à trois éléments : le label, dans un cas la CroixRouge et, dans l'autre le sans frontiérisme ; la spécificité des terrains d'intervention c'est-à-dire les zones de conflits; et enfin les philosophies fondées sur le témoignage pour MSF, et sur la neutralité et la confidentialité pour le CICR. Cela est relayé par des modes communicationnels principalement axés sur la lisibilité c'est-à-dire sur l'explicitation de ce que font ces organisations.

Les pratiques de communication mises en place sont assez variées et alternent des actions grand public avec des actions plus réticulaires, spécifiques et explicites sur les valeurs défendues. Elles favorisent la différenciation face aux parties prenantes directes par des prises de position divergentes et singulières, et la non-conformité aux regroupements, normes et essais d'homogénéisation à l'œuvre dans le secteur ces dernières années. Les relations publiques sont privilégiées même si elles restent assez contrôlées dans le cas du CICR, fervent défenseur d'une communication bilatérale et confidentielle en rapport avec son identité. De son côté, MSF multiplie les communiqués de presse et témoigne sur les terrains qu'elle connaît avec comme objectif sauvegarder sa philosophie de dénonciation. Dans ces deux structures, les directions de la communication sont totalement intégrées de façon matricielle à l'ensemble du dispositif institutionnel et ses actions viennent en soutien aux opérations, « le cœur de la maison ». Force est de constater d'ailleurs que les acteurs, dans l'ensemble, ont une bonne perception de leurs pratiques de communication et ne dénoncent pas d'incohérences majeures ou de divergences importantes avec l'objet organisationnel.

Ces tendances semblent favoriser le sentiment d'avoir une organisation assez légitime malgré les secousses environnementales et surtout une identité reposant sur des valeurs centrales, distinctives et continues fortes. Cette identité correspond à ce qui est véhiculé par les actions de communication très différenciantes face à des publics spécifiques. A ce stade, les acteurs semblent privilégier le fait d'avoir une image plutôt positive et forte, mais accordent peu d'importance au fait qu'elle soit connue de tous les publics. Les acteurs se reconnaissent dans ce qui est diffusé car cela correspond à leur identité et ils semblent, de ce fait, moins inquiets quant à l'image qu'a le grand public en général. Cela est d'autant plus facilité par une légitimité relativement acquise. Dans le cas du CICR, elle repose sur un mandat légal, des ressources nécessaires pour travailler, et des normes d'intervention en accord avec ce qu'on attend de lui. Pour MSF, c'est le résultat d'un positionnement fort sur un secteur dont elle est pionnière. Tous ces paramètres semblent contribuer à la 


\section{Dossier : Retour sur les images d'organisations}

congruence solide entre l'image et l'identité, encore favorisée par des pratiques de communication plus orientées sur le maintien de cette identité. Les acteurs perçoivent un alignement entre l'image et l'identité organisationnelles.

\section{Une communication dissonante?}

A l'inverse, ACF et HI Belgique expriment plus d'inquiétudes. Non seulement les membres de ces deux associations perçoivent leur image comme confuse dans l'esprit du public, mais ils se voient aussi souffrir d'un déficit de reconnaissance spontanée, d'une image erronée, voire même peu valorisante. Les caractéristiques observées comme exprimées semblent pouvoir offrir des débuts de réponses à cet état de fait.

Les acteurs interrogés expriment un sentiment assez critique à l'encontre des pratiques communicationnelles mises en place jusqu'alors. Ces actions issues de directions à part entière, mais souvent en marge de l'ensemble du reste des structures, sont assez concentrées sur l'obtention d'une certaine légitimité perçue comme pas encore véritablement acquise. En effet, dans l'un comme dans l'autre cas, le déséquilibre entre les fonds publics prédominants et les fonds privés encore trop insuffisants met les organisations dans un certain inconfort et dans une inquiétude permanente quant à l'avenir. Cela semble encore renforcé par une image inadéquate voire négative. On note un certain auto-entraînement pénalisant entre le manque de légitimité et une image défavorable.

Ce mécanisme semble trouver ses sources dans des pratiques de communication très axées sur l'obtention de la légitimité avec des actions en accord avec les attentes du public. La communication est faite sur une partie de l'activité plus «attractive» pour l'opinion publique comme les mines pour HI ou la famine pour ACF. Des opérations marketing multiples sont chargées d'obtenir plus de soutien et d'élargir le cercle des donateurs. Des rapprochements avec les parties prenantes directes sont faits par le biais de la participation à des plateformes communes et la mise en place d'une stratégie légitimante à travers la recherche de la conformité (Di Maggio et Powell,1983) ${ }^{16}$. Dès lors, cela génère une structure hybride entre ce

16 Di Maggio P.J., Powell W.W., The Iron Cage Revisited: Institutional Isomorphism and Collective Rationality in Organizational Fields, American Sociological Review, vol. 48, 1983, pp. 147-160. 
qui est vécu en interne et ce qui est véhiculé, à travers cette image, à l'extérieur.

Enfin, les personnes interrogées invoquent une certaine remise en question de leur identité du fait de la difficulté à trouver des valeurs distinctives (différentes face aux autres), et continues (pérennes dans le temps). Ce dysfonctionnement est sous-tendu par une inadéquation entre l'image véhiculée totalement parcellaire voire tronquée, et une identité décalée et peu valorisée qui tend à essayer de s'ajuster, parfois à ses dépens, au monde qui l'entoure et à ce qu'on attend d'elle. Cela semble expliquer, en grande partie, la mauvaise image que ces acteurs ressentent de la part de leur environnement. Les pratiques de communication axées sur l'obtention de la légitimité peuvent, si elles ne sont pas contrebalancées par d'autres pratiques non seulement déstabiliser l'identité de l'organisation par la diffusion d'une image inadéquate, mais aussi générer un certain nombre de dichotomies au sein de l'organisation notamment entre l'objet communicationnel et l'objet organisationnel, entre sa configuration interne et sa représentation externe. Cela est d'autant plus dangereux que les acteurs clés ressentent cette dissonance.

Malgré une présentation volontairement succincte des résultats de cette étude, ces quatre organisations semblent proposer deux modèles communicationnels générateurs de deux configurations identitaires. D'un côté, face à une communication orientée sur l'identité, l'image véhiculée comme l'image ressentie par les membres de ce que le public pense semblent en adéquation. Cela renforce encore non seulement l'identité organisationnelle, mais aussi continue à asseoir une certaine légitimité. De l'autre côté, une communication très focalisée sur l'obtention de cette légitimité a tendance à découpler l'image véhiculée à l'extérieur par rapport à l'identité construite en interne. Cela génère une structure hybride avec non seulement un affaiblissement de cette identité, mais aussi un mauvais ressenti de l'image perçue par l'extérieur, dévalorisante pour la perception de l'assise sociétale de l'organisation et du bien-fondé de sa légitimité.

Pour véritablement vérifier ces corrélations, ces premiers résultats méritent d'être encore approfondis d'un point de vue conceptuel et élargis par leur application éventuelle à un corpus plus étendu. Cependant, ils se veulent heuristiques et sont posés comme les prémisses d'une étude plus développée, en œuvre actuellement, tentant de décrypter les phénomènes corrélatifs entre ces éléments si stratégiques au sein des organisations. 
Dossier : Retour sur les images d'organisations

\section{Bibliographie}

ALBERT S.; WHETTEN D.A., Organizational Identity, Research in Organizational Behaviour, vol. 7, L.L. Cummings \& B.M. Staw, Eds Greenwich, CT, JAI, 1985, pp. 263-295.

ARDOINO J., PAULRE B., Groupes, relations et communication, Dictionnaire Critique de la Communication (sous dir.) L. Sfez, Tome 1, Paris : PUF, pp. 602-618.

BERNARD F., Communication organisationnelle et usages des sciences humaines et sociales. Fragments de réflexion. Communication d'entreprises et d'organisations, Textes réunis par C. Le Moenne), Rennes : PUR, 1998, pp. 48-73.

CORLEY K.G., GIOIA D.A., "Identity Ambiguity and Change in the Wake of a corporate spin-off", in Administrative Science Quarterly, $\mathrm{N}^{\circ} 49,2004$, pp 173-208.

DEPELTEAU F., La démarche d'une recherche en sciences humaines, Laval : Les Presses de l'Université de Laval, 2002, p. 214.

DI MAGGIO P.J., POWELL W.W., "The Iron Cage Revisited: Institutional Isomorphism and Collective Rationality in Organizational Fields", in American Sociological Review, vol. 48, 1983, pp. 147160.

DUTTON J.E., DUKERICH J.M., HARQUAIL C.V., " Organizational images and member identification", in Administrative Sciences Quarterly, 39, 1994, pp. 239-263.

GIROUX N., L'étude de cas, Conduire un projet de recherche - une perspective qualitative, Y. Giordano (Dir.), Colombelles : Editions EMS, 2003, pp. 42-84.

GIROUX N., «La communication interne : une définition en évolution », in Communication \& Organisation $\mathrm{n}^{\circ} 5, \quad \mathrm{La}$ communication interne : une approche croisée, GREC/O, 1994.

KAPFERER J.-N.., « Maîtriser l'image de l'entreprise : le prisme de l'identité », in Revue Française de Gestion, Nov-Déc. 1988, pp : 7882.

LEGENDRE P., Leçon III- Dieu au miroir, Etude sur l'institution des images, Paris, Fayard, 1994.

MARION G., Les images de l'entreprise, Paris: Les Editions d'Organisation, 1989. 
L'image des organisations humanitaires...

MAURER J.G., Readings in organizational theory: open-system approaches, New York, random House, 1971.

PRATT M.G., FOREMAN P.O, "Classifying Managerial responses to multiple Organizational identities," in Academy of Management Review, vol. 25, n 1,2000 , pp. 18-42.

SUCHMAN M., "Managing Legitimacy: Strategic and Institutional Approaches", in Academy of Management Review, Vol. 20, n³, 1995, pp. 571-610.

WHETTEN A.E., ALBERT and WHETTEN "revisited-Strenghtening the concept of organizational identity", in Journal of Management Inquiry, vol. $15 \mathrm{n}^{\circ} 3$, September 2006, pp. 219-234. 